\title{
一种热解炭在金属钠中的相变
}

\author{
徐子颕 ${ }^{*}$ 吉 涛 王玮衍 夏炳忠 马 超 甘礼华 \\ (同济大学化学系, 上海 200092)
}

\begin{abstract}
摘要: 通过酚醛树脂的裂解和碳化所形成的热解炭与金属钠在氩气保护气氛中加热, 得到一种无定形碳在常 压和较低温度下进行石墨化的方法, 并研究了热解炭在金属钠熔体中的相变. 对所得样品用 X射线粉末衍射 (XRD)、光散射拉曼光谱、透射电子显微镜(TEM)以及Brunauer-Emmett-Teller (BET)法氮气吸附进行表征与分 析. 结果表明: 热解炭在金属钠熔体中于 $800{ }^{\circ} \mathrm{C}$ 加热 $24 \mathrm{~h}$, 发生明显的石墨化; 于 $900{ }^{\circ} \mathrm{C}$ 加热 $24 \mathrm{~h}$, 所得样品 的石墨化度为 $40 \%$, 石墨化碳的平均厚度约为 $40 \mathrm{~nm}$, 孔结构由微孔转变为介孔. 探讨了金属钠在无定形碳中 的渗透扩散导致其相变的原因.
\end{abstract}

关键词: 酚醛树脂; 热解炭; 石墨化; 金属钠; 相变 中图分类号: $0642 ; 0792$

\section{Phase Transformation of Pyrocarbon in Molten Sodium Metal}

\author{
XU Zi-Jie* JI Tao WANG Wei-Yan XIA Bing-Zhong MA Chao GAN Li-Hua \\ (Department of Chemistry, Tongji University, Shanghai 200092, P. R. China)
}

\begin{abstract}
A method to graphitize amorphous carbon was carried out by annealing pyrocarbon from cracked phenolic resin in molten sodium metal at a lower temperature and ambient pressure and the phase transformation of pyrocarbon from amorphous carbon to crystallized carbon was studied. X-ray diffraction (XRD), Raman scattering spectroscopy, transmission electron microscopy (TEM), and nitrogen gas physisorption by the Brunauer-Emmett-Teller (BET) method were used to probe the prepared samples for carbon composition, particle size, and morphology. The graphitization of amorphous carbon was obvious when being annealed in molten sodium metal in argon atmosphere at $800^{\circ} \mathrm{C}$ for $24 \mathrm{~h}$. For the sample annealed at $900{ }^{\circ} \mathrm{C}$ for $24 \mathrm{~h}$, the degree of graphitization was $40 \%$ and the average thickness of the graphitized carbon layers was about $40 \mathrm{~nm}$. The effect of sodium metal infiltration into the matrix of amorphous carbon on the graphitization is also discussed.
\end{abstract}

Key Words: Phenol resin; Pyrocarbon; Graphitization; Sodium metal; Phase transformation

炭/炭复合材料是一种多相非均质混合物, 因其 具有高比强度、高比模量等显著的材料结构性能, 逐 渐成为新一代航空航天材料的发展方向. 然而炭/炭 复合材料的石墨化, 会影响该类材料的力学性能、物 理性能和化学性能, 是最重要的结构控制因素之一, 通过调整该类材料的石墨化状态, 可改善其综合性 能, 从而满足不同的使用要求. 因此, 开展无定形碳 材料在较低温度下的石墨化研究对炭/炭复合材料
的应用具有重要的意义.

无定形碳的石墨化就是在一定的二维平面范 围内有序的乱层结构碳的残片进行定向重排的相 变过程. 由于在该相变过程中, 无定形碳容易形成 亚稳态, 使得这种相变的阻力增大, 因此商品化石 墨的生产一般都在 $2700{ }^{\circ} \mathrm{C}$ 左右进行. 但是, 在如此 高温条件下进行石墨化, 使得材料的力学和电学性 能受到损害, 如无定形碳材料在 $2700{ }^{\circ} \mathrm{C}$ 经石墨化

Received: August 9, 2010; Revised: September 12, 2010; Published on Web: November 15, 2010.

"Corresponding author. Email: xuzijie-tj@126.com; Tel: +86-21-65982654-8430

(C) Editorial office of Acta Physico-Chimica Sinica 
所得样品的放电容量为 $74 \mathrm{mAh} \cdot \mathrm{g}^{-1}$, 而在 $1000{ }^{\circ} \mathrm{C}$ 温度条件下石墨化后, 所得样品的放电容量为 250 $\mathrm{mAh} \cdot \mathrm{g}^{-1[1]}$. 目前, 基于溶解再析出和碳化物转化机 理的催化石墨化方法可以有效地降低石墨化温度, 具体方法主要有两类: 其一, 在碳基质中加入过渡 金属及其氧化物, 如 $\mathrm{Fe} 、 \mathrm{Mn} 、 \mathrm{Cr}$ 等过渡金属及其氧 化物 ${ }^{[2-3]}$; 其二, 在碳基质中形成三组分的插层化合 物, 如 Tanaike 等 ${ }^{[4]}$ 将金属 $\mathrm{Li} 、 \mathrm{Na}$ 和 $\mathrm{K}$ 溶于四氢呋喃 中, 获得相应的有机金属化合物, 结果表明所得材 料的石墨化度很低. Rojas-Cervantes ${ }^{[5]}$ 和 Oya ${ }^{[6]}$ 等合 成了分别含有金属 $\mathrm{Na} 、 \mathrm{~K} 、 \mathrm{Mg}$ 和 $\mathrm{Zr}$ 的碳的干凝胶, 在 $1000{ }^{\circ} \mathrm{C}$ 氮气气氛中烧结, 没有发现这些金属的 催化活性, 得到的仍然是无定形碳.

由于酚醛树脂产碳量高, 常被用作制备先进碳 材料的先驱物. 选用酚醛树脂类物质作为碳源, 经热 解后得到热解炭, 开展其石墨化的研究近年来已引 起人们的重视. 张福勤等 ${ }^{[7]}$ 研究了化学气相沉积热 解炭的可石墨化性; 王永刚等 ${ }^{[8]}$ 采用化学气相渗透 对泡沫碳进行复合处理, 在 $2500{ }^{\circ} \mathrm{C}$ 得到石墨化泡 沫碳; 周德风等 ${ }^{[9}$ 报道了在酚醛树脂中加入氯化锌, 可以改变热解炭的微观结构及石墨化程度; Chen 等 人 ${ }^{[10-11]}$ 使用硝酸镨作催化剂研究其对酚醛树脂热解 炭的石墨化作用, 在催化剂含量为 $15 \%(w)$ 以及 $2400{ }^{\circ} \mathrm{C}$ 时获得最优化的石墨化条件, 他们还用含量 为 $29 \%(w)$ 的石墨氧化物作催化剂在 $2400{ }^{\circ} \mathrm{C}$ 时获 得较完整的石墨结构; $\mathrm{Cai}$ 等 ${ }^{[12]}$ 使用含量为 $5 \%(w)$ 的 铁镍催化剂, 在外加磁场以及 $1200{ }^{\circ} \mathrm{C}$ 时实现酚醛 树脂的石墨化. 除此之外, 还有文章报道 ${ }^{[13]}$ 使用金属 钇作催化剂研究酚醛树脂的催化石墨化.

本文通过将酚醛树脂裂解和碳化后形成的热解 炭与金属钠在氩气保护下加热, 开展无定形碳在金 属钠熔体中的相变研究. 采用 X射线粉末衍射 (XRD) 以及激光散射拉曼光谱技术, 对所得样品碳 组成的相态以及层内、层间碳原子的状态进行表 征; 通过透射电子显微镜(TEM)观察碳组成的形貌, 通过比表面积分析研究热解炭在石墨化前后孔结构 特征的变化. 探讨了金属钠在无定形碳基质中的渗 透与扩散对无定形碳相变产生的影响. 该方法可用 于新型结构的炭/炭复合材料的石墨化研究中.

\section{1 实 验}

\section{1 热解炭的制备}

将市售酚醛树脂(2130型, 无锡久耐防腐材料有
限公司)放入烘箱(102A-2 型, 上海试验仪器总厂) 中, 调节温度到 $80^{\circ} \mathrm{C}$, 保温 $10 \mathrm{~h}$, 再升温至 $120^{\circ} \mathrm{C}$, 保温 $10 \mathrm{~h}$, 继续升温至 $140^{\circ} \mathrm{C}$ 并保温 $24 \mathrm{~h}$, 使酚醛树 脂完全固化. 将固化后的酚醛树脂放入管式炉 (SK2-15-13T 型, 上海实验电阻炉厂) 中, 通入氩气保 护, 以 $10^{\circ} \mathrm{C} \cdot \mathrm{min}^{-1}$ 的升温速率升温至 $200^{\circ} \mathrm{C}$, 保温 3 $\mathrm{h}$, 再以相同的升温速率升温至 $800^{\circ} \mathrm{C}$ 并保温 $4 \mathrm{~h}$, 得 到热解炭.

\section{2 石墨化方法}

称取 $5 \mathrm{~g}$ 按上述方法制备的热解炭, 放入带盖的 坩埚中, 在充有氩气的手套箱(ZKX1 型, 南京南大 仪器厂)内切割金属钠块, 并称取 $3 \mathrm{~g}$ 放置其表面, 再 将坩埚置于管式炉(SK2-15-13T 型, 上海实验电阻 炉厂)中并通入氩气保护, 以 $10^{\circ} \mathrm{C} \cdot \mathrm{min}^{-1}$ 的升温速率 升温至所设定温度并保温 $24 \mathrm{~h}$, 本实验所设定的温 度分别是 $600 、 700 、 800$ 和 $900{ }^{\circ} \mathrm{C}$; 将所得样品用蒸 馏水超声清洗, 直至洗液的 $\mathrm{pH}$ 值为 7 , 再将清洗后 的用品在烘箱(102A-2 型, 上海试验仪器总厂)内于 $120{ }^{\circ} \mathrm{C}$ 干燥.

\section{3 表征方法}

使用 D8 FOCUS 型X射线粉末衍射仪(德国, Bruker AXS) 对样品进行 XRD 表征, 测试条件为 40 $\mathrm{kV}, 40 \mathrm{~mA}, \mathrm{Cu} K_{a}$ 射线; 使用 Renishaw inVia激光拉 曼光谱仪(英国, Renishaw)对所得样品进行拉曼光 谱分析; 使用 S-TWIN F20 型场发射透射电镜(荷兰, FEI)对样品进行 TEM 形貌表征; 使用 Micromeritics Tristar 3000 比表面积测定仪(美国, Micromeritics), 采用 Brunauer-Emmett-Teller (BET)法分析样品的比 表面积、孔径分布以及孔结构特征.

\section{2 结果与讨论}

选用酚醛树脂作为碳源, 经 $800^{\circ} \mathrm{C}$ 热解、碳化 后得到热解炭, 用以研究无定形碳材料在金属钠熔 体中的相变. 由于残存于样品中的金属钠在样品的 后处理中遇到空气被氧化, 形成的氧化钠成分在 $\mathrm{XRD}$ 检测时会产生很强的衍射峰, 干扰了对碳组成 的表征, 因此, 样品在表征前必须经蒸馏水洗涤, 去 除氧化钠组分.

\section{1 不同温度条件下热解炭在金属钠中的相变}

图 1 是热解炭在不同温度条件下进行热处理所 得样品的 XRD 谱. 图谱(1)为在没有金属钠存在的 条件下, 将热解炭在 $900{ }^{\circ} \mathrm{C}$ 保温 $24 \mathrm{~h}$, 所得样品的碳 组成仍然是典型的无定形碳, 表明无定形碳在此温 
度时没有发生相变. 当热解炭与金属钠在 $600{ }^{\circ} \mathrm{C}$ 加 热 $24 \mathrm{~h}$, 得到谱(2), 根据文献[6]的解释, 表明金属粒 子已经渗透和扩散在无定形碳的基质中, 使得其中 的乱碳结构残片开始在局部进行重新取向, 导致 $2 \theta$ 分别在 $25^{\circ}$ 和 $45^{\circ}$ 附近出现较为明显的漫衍射峰. 当 热解炭与金属钠中的加热温度为 $700{ }^{\circ} \mathrm{C}$ 时, 得到谱 (3), 从中可见其特征衍射峰已经明显锐化, 表明此 时热解炭中的无定形碳已经晶格化. 当加热温度升 高至 $900{ }^{\circ} \mathrm{C}$, 得到谱(4), 显示石墨化碳的特征衍射 峰更加锐化. 当样品的加热温度从 $700{ }^{\circ} \mathrm{C}$ 升高至 $900{ }^{\circ} \mathrm{C}$ 时, 样品的衍射数据也相应发生变化, 其中 $2 \theta$ 值从 $25.9^{\circ}$ 增加至 $26.3^{\circ}$, 相应的 $d_{002}$ 值从 0.3433 $\mathrm{nm}$ 变为 $0.3406 \mathrm{~nm}$. 根据 Mering 和 Maire 公式 ${ }^{[14]}$, 样品的石墨化度可由 $G=\left(\left(0.3440-d_{002}\right) /(0.3440-\right.$ $0.3354)) \times 100 \%$ 计算得到, 当加热温度从 $700{ }^{\circ} \mathrm{C}$ 升高 至 $900{ }^{\circ} \mathrm{C}$ 时, 所得样品的石墨化度分别从 $8.5 \%$ 增加 至 $40 \%$. 对一系列样品的 XRD 表征结果的分析表 明, 在金属钠熔体中, 无定形碳的碳组成发生明显的 变化, 随着加热温度的升高, 热解炭的石墨化特征愈 加明显.

图 2 是所选样品的拉曼谱图. 在 1350、1570 和 $2700 \mathrm{~cm}^{-1}$ 处的谱峰被分别称为 $D 、 G$ 和 $G^{\prime}$ 峰. 图谱 中 $D$ 峰是发生于相同碳原子间的拉曼振动模式, $G$ 峰则表示两种不同碳原子之间的光子振动模式, 而 $G^{\prime}$ 峰表示一种源自晶面之间的碳原子所发生的光 子振动模式, 是一种二阶拉曼散射过程. 谱图(1)是 酚醛树脂裂解碳在没有金属钠存在的条件下, 经过 $900{ }^{\circ} \mathrm{C}$ 加热后所得样品的拉曼谱图, 图谱中 $D$ 峰强 度高于 $G$ 峰并且两峰没有完全分离, 另外, 图谱中无

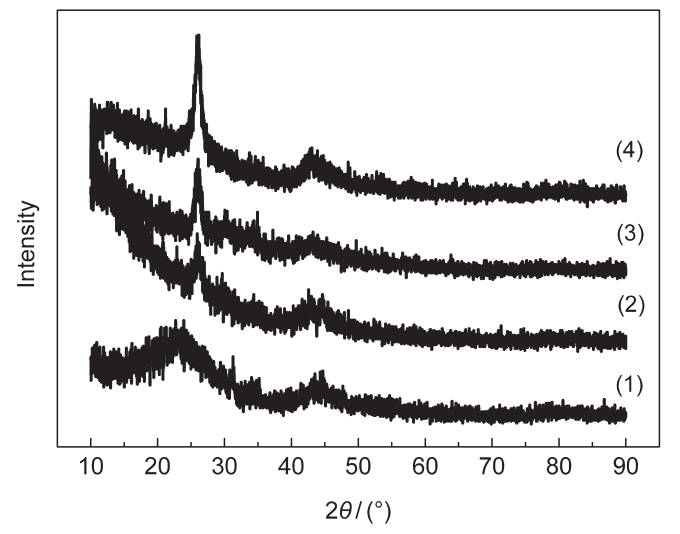

图 1 热解炭在不同条件下退火 $24 \mathrm{~h}$ 的 XRD 图谱

Fig.1 XRD patterns of pyrocarbons annealed at different conditions for $24 \mathbf{h}$

(1) $900{ }^{\circ} \mathrm{C}$ without sodium metal; (2) $600{ }^{\circ} \mathrm{C}$, (3) $700{ }^{\circ} \mathrm{C}$, and (4) $900{ }^{\circ} \mathrm{C}$ in molten sodium metal
$G^{\prime}$ 峰, 表明样品对激光的漫散射分别在乱碳结构残 片内部的碳原子以及乱碳结构残片间进行, 这种光 子振动模式证明了热解炭中的无定形碳含有二维有 序的乱碳结构而且呈现杂乱无章地堆积. 图谱(2)是 热解炭在金属钠中, 经过 $700^{\circ} \mathrm{C}$ 加热所得样品的拉 曼谱图, 此时 $D$ 峰强度降低, $G$ 峰强度增加并呈现两 峰分离的迹象, 表明激光在样品中不同碳原子间的 光子振动模式加强. 另外, 图谱中同时呈现一个明显 的 $G^{\prime}$ 峰, 表明光子振动发生在石墨化层间的碳原子 之间, 进一步说明乱碳结构残片在此时已经发生明 显的定向重排. 图谱(3)是热解炭在金属钠中, 经过 $900{ }^{\circ} \mathrm{C}$ 加热所得样品的拉曼谱图, 此时 $D$ 峰强度明 显降低, $G$ 峰强度明显增加而且两峰完全分离, 表明 光子的振动模式主要发生在晶面之间的碳原子中, 说明该样品中的碳原子已经转变为石墨结构.

\section{2 金属钠在无定形碳中的渗透与扩散对其相变 的影响}

根据对所得样品进行的 XRD 和拉曼谱分析知, 在没有金属钠存在时, 热解炭在 $900{ }^{\circ} \mathrm{C}$ 加热条件下 没有发生相变, 而有金属钠存在的条件下, 无定形碳 在 $700{ }^{\circ} \mathrm{C}$ 加热 $24 \mathrm{~h}$ 后, 观察到无定形碳开始向结晶 态碳转化, 随着加热温度的升高, 这种相态转化更加 明显. 因此, 金属钠的存在是导致热解炭在加热条件 下发生相变的必要因素. 虽然目前对金属钠与碳组 成的相互作用机制进行原位的实时表征和分析还比 较困难, 但是, 根据XRD 和拉曼的表征结果, 不仅证 实了这种相变的发生, 而且揭示金属钠对该相变的 重要影响, 即金属钠原子在无定形碳中的渗透与扩 散引起了其中乱碳结构残片的重排与取向. 当金属

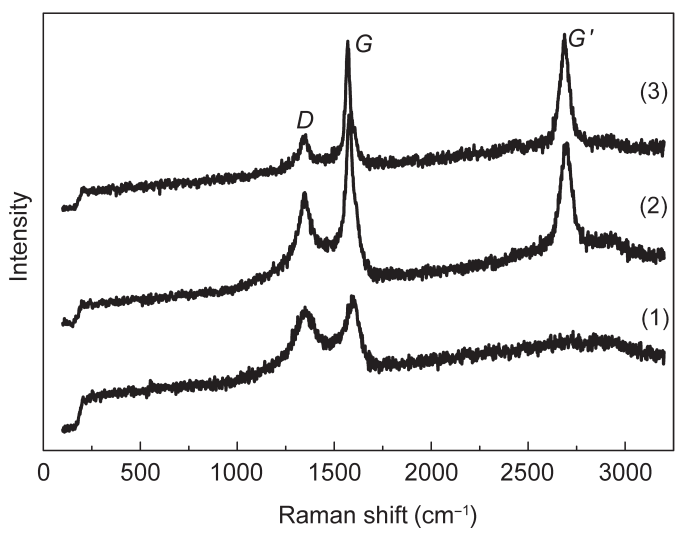

图 2 热解炭在 $900{ }^{\circ} \mathrm{C}$ (1)以及在金属钠中于 $700{ }^{\circ} \mathrm{C}$ (2) 和 $900{ }^{\circ} \mathrm{C}(3)$ 加热 $24 \mathrm{~h}$ 的拉曼图谱

Fig.2 Raman spectra of pyrocarbons annealed at $900{ }^{\circ} \mathrm{C}$ without sodium metal (1) and at $700{ }^{\circ} \mathrm{C}(2), 900{ }^{\circ} \mathrm{C}$

(3) in molten sodium metal for $24 \mathrm{~h}$ 
钠原子渗入到乱碳结构残片间, 钠原子外层电子的 高活泼性, 影响了残片中碳原子周围的电场环境, 同 时渗透与扩散在其中的钠原子可以形成金属钠的连 续相, 并充当优良的导热介质, 使得无定形碳在石墨 化过程中的结晶潜热能够通过导热介质及时地向周 围环境释放, 从而有利于无定形碳在较低温度条件 下发生相变. 金属钠在无定形碳中的渗透、扩散与加 热温度密切相关. 随着加热温度的升高, 对所得样品 的 XRD 和拉曼表征结果都表现出了高度的一致性, 即石墨化度增加. 无定形碳在金属钠中的相变过程 示意图如图 3 所示.

对样品形貌学的观察进一步证实了金属钠对热 解炭在其中发生相变的影响. 图 4(a) 是热解炭与金 属钠在 $700^{\circ} \mathrm{C}$ 加热 $24 \mathrm{~h}$ 所得样品的 TEM 照片, 图中 “A”所在区域为无定形碳, “ $\mathrm{T}$ ”所在区域显现出湍流 碳的形貌特征, “G”区域则显现出石墨化碳的形貌 特征. 通过对样品不同区域进行 TEM 观察, 发现湍 流碳总是出现在无定形碳和石墨化碳的过渡区域, 其形貌特征显示出无定形碳中的乱碳结构残片已在 有限范围内进行了定向重排, 但不够完整. 在 $700{ }^{\circ} \mathrm{C}$ 加热条件下, 金属钠在无定形碳中的渗透与扩散不 完全, 没有形成均匀的金属钠连续相, 使得乱碳结构 残片的定向重排过程不能在长程范围内连续进行. 湍流碳的出现使得无定形碳向石墨化碳转化的阻力 增加, 这也是商品化石墨必须在高温条件下生产的 主要原因.

图 4(b) 是热解炭与金属钠在 $900{ }^{\circ} \mathrm{C}$ 加热 $24 \mathrm{~h}$ 所 得样品的 TEM 照片, 随着加热温度的升高, 石墨化 碳的厚度与长度显著增加, 所得样品中石墨化碳的 平均厚度约为 $40 \mathrm{~nm}$, 显然升高温度加速了金属钠 在碳基质中的渗透与扩散, 有利于无定形碳向石墨 化碳的转化.

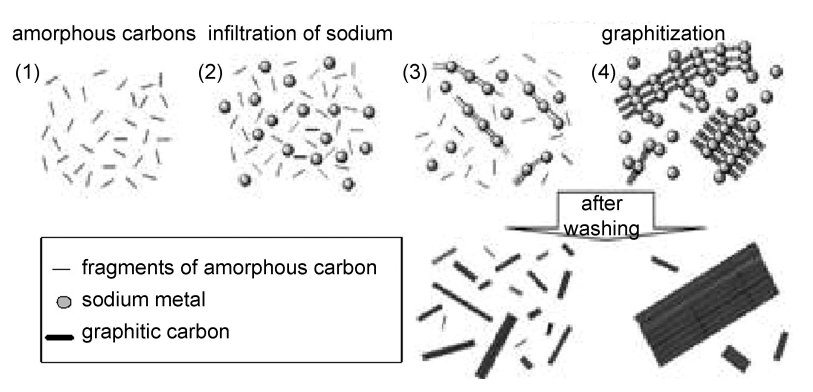

图 3 无定形碳在金属钠中的相变过程示意图

Fig.3 Sketch map of the phase transformation of amorphous carbons in molten sodium metal The conditions of (1)-(4) are the same as those in Fig.1.
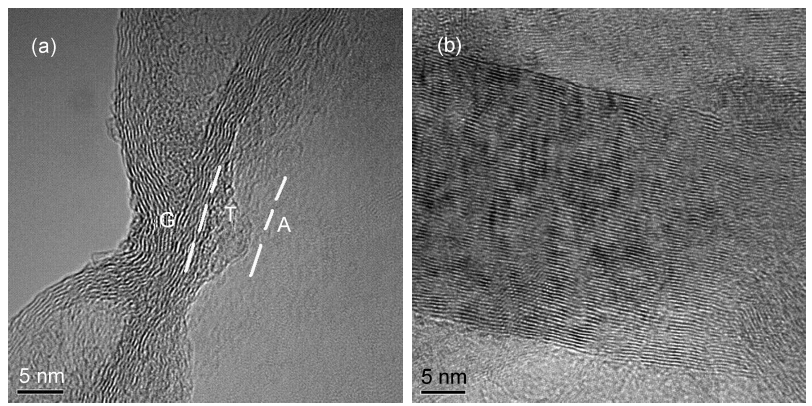

图 4 热解炭与金属钠在 700 (a)和 $900{ }^{\circ} \mathrm{C}(\mathrm{b})$ 加热 $24 \mathrm{~h}$ 所得 样品的 TEM照片

Fig.4 TEM image of pyrocarbons annealed in molten sodium metal at $700{ }^{\circ} \mathrm{C}$ (a) and $900{ }^{\circ} \mathrm{C}$ (b) for $24 \mathrm{~h}$

Area A denotes amorphous carbons, aera $\mathrm{T}$ denotes turbostratic carbons, and area $\mathrm{G}$ denotes graphitic carbons.

\section{3 在金属钠作用下热解炭中孔结构特征的变化}

图 5 是热解炭在金属钠中发生相变前后样品的 对氮气的吸附-脱附等温线. 从图中可见, 热解炭样 品的吸附-脱附等温线属于类型 I, 插图所示的孔分 布曲线表明热解炭中存在大量孔径小于 $2 \mathrm{~nm}$ 的微 孔, 等温线中很小的滞后环表明热解炭中的微孔对 氮气的吸附与脱附具有良好的可逆性, 这些孔结构 特征反映出热解炭中的微孔分布在无定形的乱碳结 构残片之间并具有良好的连通性. 然而当热解炭与 金属钠在 $900{ }^{\circ} \mathrm{C}$ 加热 $24 \mathrm{~h}$ 所得石墨化热解炭样品 的氮气吸附-脱附等温线出现一个较大的滞后环(如 等温线 2 所示), 插图中的孔分布曲线显示样品中的 孔径尺寸主要集中在 4-5 nm 之间, 属于介孔尺寸, 表明热解炭在石墨化前后孔结构发生由微孔向介孔 发生转变. 导致孔结构转化的原因是由于热解炭中

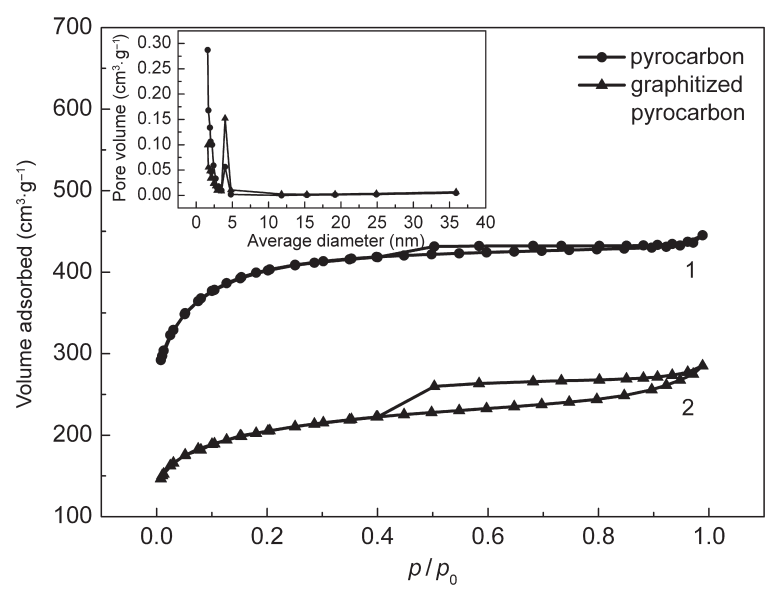

图 5 样品的氮气吸附-脱附等温线

Fig.5 Nitrogen adsorption desorption isotherms of selected samples

Isotherm 1 represents the sample of pyrocarbon and isotherm 2 represents the sample of graphitized pyrocarbon. Inset is pore size distribution curve. 
的乱碳结构残片在金属钠原子的作用下定向重排, 使得原先分布在其中的相互连通的微孔发生合并增 大, 形成了分布在石墨化碳层间的插层状孔, 由于此 时毛细管作用力的增强, 导致样品对氮气的脱附滞 后. 另外, 从图中可见, 热解炭发生石墨化后, 随着乱 碳结构残片的定向重排使得石墨化热解炭样品的比 表面积有所下降. 酚醛树脂热解炭在金属钠作用下, 其孔结构特征的改变是碳组成发生相变的结果, 同 时也进一步证实了金属钠原子在乱碳结构残片间的 渗透与扩散有利于其发生定向重排, 从而导致热解 炭能够在较低的温度条件下实现石墨化.

催化石墨化是通过在碳基质中引入催化剂, 以 降低石墨化温度, 但是, 目前的方法对于一些新型碳 材料的石墨化显现出一定的缺陷. 首先, 在材料的制 备阶段必须将催化剂加到碳材料的基质中, 这势必 增加了材料制备的难度, 甚至对材质特性产生不良 影响. 例如, 对碳气凝胶材料的石墨化, 如果采用现 有的催化石墨化方法, 就需要在碳气凝胶制备所必 经的溶胶-凝胶过程中加入催化剂, 它可能改变胶体 离子的微环境, 继而影响了碳气凝胶的结构特性. 其 次, 目前所采用的催化剂多数是过渡金属的氧化物, 在碳材料石墨化以后, 很难将催化剂从碳材料的基 质中去除干净, 这可能对转型后碳材料的电学特性 或电化学催化特性等产生影响. 再者, 现有催化石墨 化方法 ${ }^{[4-6]}$ 对于碳气凝胶的石墨化效果仍不理想. 酚 醛树脂热解炭在金属钠作用下发生相变进行石墨 化, 该方法不仅具有操作简单的特点, 而且可以避免 在碳基质中加入催化剂给材质纯度、特性带来的不 利影响并且可以降低材料的制备难度. 另外, 该方法 有利于一些新型多孔性碳材料的石墨化, 因为多孔 性结构十分有利于金属钠在碳基质中的渗透与扩 散. 而实现这类材料的低温石墨化, 可以使无定形碳 材料的多孔特性与石墨晶体材料的材质特性相结 合, 有助于扩展碳材料在传感器、探测器、航天以及 新能源电池等领域的应用范围. 我们以自制的碳气 凝胶通过文中方法进行石墨化研究, 初步结果表明 在 $800{ }^{\circ} \mathrm{C}$ 实现了碳气凝胶的石墨化, 相关研究工作 正在进行中.

\section{3 结 论}

由酚醛树脂经过裂解碳化后得到的热解炭, 通
过与金属钠一起在氩气保护下, 在 $800{ }^{\circ} \mathrm{C}$ 加热 $24 \mathrm{~h}$ 可以观察到明显的石墨化现象. 金属钠在无定形碳 中的渗透与扩散引起乱碳结构残片的定向重排, 湍 流碳的形成是金属钠在其中的渗透与扩散不均匀所 致, 是无定形碳向石墨化碳转化的中间相态, 通过升 高加热温度, 可以改善金属钠在其中的扩散, 从而提 高了石墨化程度. 热解炭在金属钠作用下发生石墨 化使得样品中的孔结构由微孔转化为介孔, 在 $900^{\circ}$ C 时石墨化度达到 $40 \%$, 样品中石墨化碳层的平均 厚度达到 $40 \mathrm{~nm}$.

致谢：本文实验研究过程中的部分分析测试工作得到同济 大学化学系实验中心的支持.

\section{References}

1 Skowroński, J. M.; Knofczyński, K.; Inagaki, M. Solid State Ionics, 2007, 178: 137

2 Oya, A.; Otani, S. Carbon, 1979, 17: 131

3 Curtis, B. J. Carbon, 1966, 4: 483

4 Tanaike, O.; Inagaki, M. Carbon, 1997, 35: 831

5 Rojas-Cervantes, M. L.; Alonso, L.; Díaz-Terán, J.; López-Peinado, A. J.; Martín-Aranda, R. M.; Gómez-Serrano, V. Carbon, 2004, 42: 1575

6 Oya, A.; Mochizuki, M.; Otani, S.; Tomizuka, I. Carbon, 1979, 17: 71

7 Zhang, F. Q.; Huang, Q. Z.; Zou, L. H.; Huang, B. Y.; Xiong, X.; Zhang, C. F. Journal of Inorganic Materials, 2004, 19(5): 1118 [张福勤, 黄启忠, 邹林华, 黄伯云, 熊 翔, 张传福. 无机材料学 报, 2004, 19(5): 1118]

8 Wang, Y. G.; Lin, X. C.; Yang, H. J.; Zhang, J. S.; Xu, D. P. Journal of Materials Science \& Engineering, 2008, 26(3): 365 [王永刚, 林雄超, 杨慧君, 张江松, 许德平. 材料科学与工程学报, 2008, 26(3): 365]

9 Zhou, D. F.; Xie, H. M.; Zhao, Y. L.; Wang, R. S. Journal of Functional Material, 2005, 36(1): 83 [周德风, 谢海明, 赵艳玲, 王荣顺. 功能材料, 2005, 36(1): 83]

10 Yi, S. J.; Chen, J. H.; Xiao, X.; Liu, L.; Fan, Z. J. Rare Earths, 2010, 28(1): 69

11 Yi, S. J.; Chen, J. H.; Li, H. Y.; Liu, L.; Xiao, X.; Zhang, X. H. Carbon, 2010, 48: 912

12 Xu, S. H.; Zhang, F. Y.; Kang, Q.; Liu, S. H.; Cai, Q. Y. Carbon, 2009, 47: 3233

13 Ni, Z. C.; Li, Q. T.; Yan, L.; Gong, I. L.; Zhu, D. Z. Carbon, 2008, 46: 365

14 Zou, L. H.; Huang, Q. Z.; Zou, Z. Q. Carbon(China), 1998, 93(1): 8 [邹林华, 黄启忠, 邹志强. 炭素, 1998, 93(1): 8] 